\title{
A 4-week resistance training intervention improves stability, strength and neuromuscular activity in the lower limb: A case study of a cerebral palsy adult
}

\author{
Arran McManus, Matthew Bembridge, James Scales, Jonathan D Wiles, Damian A Coleman and Jamie M O'Driscoll* \\ School of Human and Life Sciences, Canterbury Christ Church University, Kent, CT1 1QT, UK
}

\begin{abstract}
Spastic diplegia, a form of cerebral palsy, is a neurological disorder that results in some degree of spasticity. It is often associated with impairment of both normal and selective movement of the lower limbs, and some degree of muscular weakness. Resistance training is an established exercise intervention with numerous neurological benefits. Evidence suggests that resistance training can positively improve stability in young populations with cerebral palsy. However, little research exists in adult populations, who are at greater risk of inactivity due to ageing and an increase in sedentarism. A single-subject (aged 35-years old, 150.5 centimetres, 81 kilograms) case-study was therefore carried out to investigate the effectiveness of a 4-week resistance training intervention on stability, neuromuscular activity and force output in a male adult with cerebral palsy. The variables measured were total centre of pressure displacement, peak torque in both the quadriceps and hamstrings and electromyography (EMG) activity in the rectus femoris (RF), vastus lateralis (VL), biceps femoris (BF) and semitendinosus (ST). The results demonstrated that a 4-week resistance training intervention reduced total centre of pressure displacement $(-51.34 \%)$ whilst concomitantly increased EMG output (RF left $+80 \%$ right $+22 \%$, VL left $+160 \%$ right $+26 \%$, BF left $+203 \%$ right $+44 \%$, ST left $+40 \%$ right_90\%) and peak torque values (right leg extension $-7 \%$, right leg flexion $+29 \%$, left leg extension $+11 \%$, left leg flexion $+42 \%$ ). In conclusion, this case study demonstrates that a 4 -week resistance training intervention improves the stability of the participant, possibly through neurological adaptations and improvements to lower limb strength.
\end{abstract}

\section{Introduction}

Cerebral palsy $(\mathrm{CP})$ is a chronic, non-progressive condition that affects the ability of the central nervous system (CNS) to regulate motor unit action potentials (MUAPs). It is categorized as a neurological disorder, caused by injury or abnormal development of the cerebellum located in the brain. Spastic diplegia is a prevalent form of CP accounting for seven to eight percent of cases [1]. It is associated with rigid limbs and spasticity, caused by perpetual firing of nerve impulses. This classification is characterised by an impairment of the upper motor neuron and its subsequent pathway via the corticospinal tract, which results in impairments in muscle fibre innervation of the distal extremity muscles $[2,3]$. These impairments may result in decreased strength and stability, subsequently increasing the likelihood of falls. risk. Due to this increase in fall risk, interventions aimed at improving strength and stability should be considered.

There is evidence to suggest that the early phases of resistance training provide adaptations to the nervous system, as a means of improving muscle strength and motor control [4]. Moreover, increasing peak torque of lower limb muscle groups is frequently shown to improve postural stability in both healthy, and patient populations. Orthopaedic efforts to reduce spasticity, through the elimination of problematic nerve routes within the spinal cord (selective dorsal rhizotomy) and the use of botulinum toxin injections, have emphasized the role of muscular weakness and inhibited motor control that exists in childhood cases of $\mathrm{CP}[5,6]$. Because of this, resistance training is considered one of the few interventions available to improve muscular strength and motor control in children [3,7] without adverse effects [8].

A substantial amount of literature exists in the cases of CP in younger children, with greater effect sizes for children below aged seven- years $(\mathrm{d}=1.430)$ compared to those above thirteen-years $(\mathrm{d}=0.931)$ [9]. However, the various growth spurts experienced throughout childhood makes improvements difficult to fully quantify. Whether motor capacity can be increased or restored in adults neglected of treatment is still unclear [1]. Further to this, there is a distinct lack of literature around more severe cases of CP (i.e. spastic triplegia, and spastic quadriplegia), and how resistance programmes could be adapted to suit those with extremely low levels of gross motor control $[5,9,10]$. The limited evidence and reduced clinical support in adult $\mathrm{CP}$ patients direct the need for investigations of alternative methods such as resistance training, to improve stability, muscular strength and motor control with the intent to reduce falls-risk and enhance day-to-day functioning.

This study aims to examine the effects of a 4-week resistance training program using seated quadricep and hamstring exercises (8 Sessions, 3 Sets of 10 repetitions, at self-perceived 75\% 1Rep max) on stability in a 35-year-old untrained cerebral diplegic male to reduce fall-risk. Secondarily it aims to examine any possible mechanistic explanations of these changes through electromyography and isometric torque analysis. The findings of this study will inform the decision to progress with further randomised clinical trials in an adult $\mathrm{CP}$ cohort.

${ }^{\star}$ Correspondence to: Jamie O'Driscoll, School of Human and Life Sciences, Canterbury Christ Church University, North Holmes Road, Canterbury, Kent, CT1 1QU, UK, E-mail: jamie.odriscoll@canterbury.ac.uk

Key words: cerebral palsy, motor unit recruitment, neural adaptations, spastic diplegia

Received: July 02, 2018; Accepted: July 16, 2018; Published: July 31, 2018 


\section{Method}

\section{Experimental Approach to the problem}

This study was based around a single subject A-B design [11], spanning across a six-week period (week 1 pre-assessment, week 2-5 intervention, week 6 post-assessment). During the intervention, the participant was required to complete two resistance training sessions per week, with a three-day recovery between sessions [12].

Each session consisted of 3 sets of 10 repetitions of leg extension and leg flexion about the knee joint, at a velocity pre-set to 45 degrees per second. The intensity for the intervention was set at 75 percent of the subject's repetition maximum; this was calculated via the maximum voluntary isometric contraction (MVIC) carried out during the initial pre-assessment.

\section{Subject}

An untrained male (aged 35 years old, 150.5 centimetres, 81 kilograms) classified as spastic diplegic with no experience of resistance training, provided voluntary informed consent to take part in this study. The participant was required to give a detailed medical history. Ethical approval was granted by departmental ethics board at Canterbury Christ Church University. They were diagnosed with spastic diplegia from birth and classified during late childhood/adolescence as Level III on the Gross Motor Function Classification System (GMFCS) [13]. During early childhood the subject underwent bilateral hamstring tenotomy to lengthen undeveloped muscle tissue. Following this surgery to further reduce spasticity, rectus femoris transfer surgery was carried out on the left leg; a procedure involving relocation of the distal tendon of the rectus femoris from the patellar behind the knee joint to the semitendinosus [14]. This procedure was not carried out on the right leg, which has subsequently affected both stability and muscular strength, leaving the subject posturally undeveloped with significantly weaker hamstrings. The subject primary form of ambulation requires the use of crutches, and a wheelchair for longer distances. He is currently not undergoing any form of physiotherapy or resistance training intervention and is not taking any medication. Aside from his current condition, there were no other health complications, and therefore he was deemed suitable to partake in this study.

\section{Instrumentation}

Stability measurements were recorded using an RSscan pressure platform (Belgium, $1068 \mathrm{~mm} \times 418 \mathrm{~mm} \times 12 \mathrm{~mm}, 7192$ sensors). The subject was instructed to stand still on the platform with arms relaxed by their sides in a neutral position, eyes open. Four trials of 20 seconds were conducted. Centre of pressure $(\mathrm{CoP})$ data was collected at a frequency of $256 \mathrm{hz}$. Data was extracted from raw .txt files, coordinates $(\mathrm{mm})$ were then exported for analysis in Microsoft Excel. Pythagoras theorem was then used to calculate mediolateral and anteroposterior, and total displacement of the CoP.

A Biodex Isokinetic Dynamometer (System 3, Serial No. 08031543, Biodex Medical Systems Inc. USA) was used to carry out both the pre- and post-assessment maximal voluntary isometric contractions (MVIC), and the resistance training intervention. The dynamometer was fitted with a unilateral knee attachment, with the subject seated in an upright position. During the MVIC the subject was required to carry out three sets of ten-second isometric contractions, each separated by 120 -seconds rest. Maximal testing of the knee extensors was carried out at a fixed angle of 90-degrees, with the knee flexors set at a fixed angle of 135-degrees. These angles were manually aligned with the use of a fluid goniometer (MIE004). The resistance training intervention involved the participant carrying out both a unilateral isokinetic leg extension and leg flexion movement about the knee joint, with the velocity pre-set to 45 -degrees per second. All movements were carried out within the subjects' full range of motion (ROM).

A Noraxon EMG 32 Channel Telemyo DTS System (2400 G2) was used to measure muscle activation of the quadriceps (vastus lateralis, rectus femoris) and hamstrings (biceps femoris, semitendinosus) for both legs. Sites were prepared by the shaving of excess hair follicles with a disposable razor, and appropriate cleaning with the use of disposable alcohol wipe to increase adhesion and reduce impedance. Electrodes were placed on each of the sites in the direction that is parallel to the line of the muscle fibres, this was assessed through manual palpation of the muscle tissue. All electrodes were placed as standard, two centimetres apart, measured using anthropometric tape (Seca 203, HSC057). The rectus femoris placement was parallel to the muscle fibres on the anterior surface of the thigh, measured halfway between the knee and the iliac spine. For the vastus lateralis, the electrodes were placed three to five centimetres above the patella, on an oblique angle just lateral to the midline. The electrodes on the biceps femoris were placed parallel to the muscle fibres on the lateral aspect of the thigh, two-thirds of the distance between the greater trochanter and the back of the knee. Electrode placement for the semitendinosus was on the medial aspect of the thigh, three centimetres from the lateral border and half the distance from the gluteal fold, to the back of the knee. All electrode placements followed the procedures outlined from Criswell, et al. [15].

The Borg's CR10, Rate of Perceived Exertion (RPE) Scale for resistance training was utilised to gauge feedback on intensity of the training intervention. Its use within resistance training has shown to be a reliable tool when monitoring intensity, even with subjects who are not familiar with exercise or the scale itself [16]. After each set of 10 repetitions the participant was required to state their perceived level of exertion via the CR10 scale. This was to ensure the level of intensity remained as closely as possible to the 75 percent of repetition maximum; should the participant believe the level of effort is less than eight on the Borg CR10 scale, then the resistance would be adjusted by five percent.

\section{Procedures}

The subject arrived at the laboratory and was initially pre-screened for height and weight measurements. The subject was then connected to a Noraxon EMG System and asked to carry out a warm up consisting of 15-unresisted seated leg extension and leg flexion movements for both limbs on the isokinetic dynamometer. This was to ensure both EMG readings were normalized, and that the participant was fully prepared to partake in physical exercise. The participant then underwent the maximal isometric pre-assessment protocol for the quadriceps and hamstrings for both the right and left leg. This was synchronized with the EMG system to provide both peak torque and mean EMG values.

The subject then attended a total of eight sessions, over a four-week period ( 2 sessions per week), during the training intervention. The subject repeated the warm-up procedure before undertaking three sets of ten-repetitions at 45 degrees per second for both the isokinetic leg extension and flexion movements. Each set was separated by a three-minute recovery [17]. All training and assessments were carried out on the right leg first as this was the leg that had minimal surgical support. Upon completion of the intervention, all baseline tests were repeated. 


\section{Data reduction}

All EMG data was analyzed using TELEmyo (2400T G2) 'myomuscle' software. Analysis of the data resulted in full-wave rectification, with signal based on a moving average algorithm using a 0.5 seconds time window. All readings were expressed in terms of electrical activity or membrane potential in micro-volts $(\mu \mathrm{V})$. Peak membrane potentials were not analyzed due to both the untrained status of the participant and the nature of their condition [18], which has created a significant degree of variation in peak membrane potentials over the course of a given contraction.

\section{Results}

\section{Centre of pressure}

Pre-assessment centre of pressure values were $29.25 \mathrm{~mm}$ for mediolateral limit and $12.0 \mathrm{~mm}$ for anteroposterior limit. After the four-week strength training intervention, this was significantly reduced to $5.5 \mathrm{~mm}$ and $7.25 \mathrm{~mm}$ respectively. These highlight a significant reduction in deviations in both the mediolateral and anteroposterior displacements. This is highlighted by a significant reduction in total displacement, having a $51.34 \%$ improvement.

\section{Peak torque output}

During the preliminary assessment the peak torque output for the MVIC was recorded at 74.6 newton meters $(\mathrm{Nm})$ for the right leg extension, and $17.1 \mathrm{Nm}$ for the right leg flexion movement. After the four-week strength training intervention there was a decrease in peak torque for the right leg flexion $69.3 \mathrm{Nm}$, but an increase observed in the right leg flexion movement $22.1 \mathrm{Nm}$. Baseline measures for peak torque output were recorded at $62.4 \mathrm{Nm}$ for left leg extension, and $16 \mathrm{Nm}$ for the left leg flexion. Post intervention, both the left leg extension and left leg flexion increased to $79.4 \mathrm{Nm}$ and $22.7 \mathrm{Nm}$ respectively. These results are outlined in Table 1, with the percentage change (+/-\%) highlighted in yellow.

\section{EMG output}

The initial pre-assessment for the right leg extension reported EMG activity at $16.3 \mu \mathrm{V}$ for the rectus femoris and $15.9 \mu \mathrm{V}$ for the vastus lateralis. During the right leg flexion movement, the results for the

Table 1. Centre of Pressure, peak torque and mean EMG values, pre- and post-intervention during the MVIC, with percentage change

\begin{tabular}{|c|c|c|c|}
\hline & Pre & Post & Change \% \\
\hline Mediolateral Limit $(\mathbf{m m})$ & 29.25 & 5.5 & -81.19 \\
\hline Anteroposterior Limit $(\mathbf{m m})$ & 12 & 7.25 & -39.58 \\
\hline CoP Total Displacement $(\mathbf{m m})$ & 137.21 & 66.76 & -51.34 \\
\hline & & & \\
\hline Right Leg Extension $(\mathbf{N m})$ & 74.6 & 69.3 & -7 \\
\hline Right Leg Flexion $(\mathbf{N m})$ & 17.1 & 22.1 & +29 \\
\hline Left Leg Extension $(\mathbf{N m})$ & 62.4 & 79.4 & +11 \\
\hline Left Leg Flexion $(\mathbf{N m})$ & 16 & 22.7 & +42 \\
\hline & & & \\
\hline Right Rectus Femoris $(\boldsymbol{\mu V} \mathbf{)}$ & 16.3 & 19.9 & +22.1 \\
\hline Right Vastus Lateralis $(\boldsymbol{\mu} \mathbf{V})$ & 15.9 & 20 & +25.8 \\
\hline Right Semitendinosus $(\boldsymbol{\mu} \mathbf{V})$ & 7.9 & 15 & +89.9 \\
\hline Right Biceps Femoris $(\boldsymbol{\mu} \mathbf{V})$ & 3.7 & 11.2 & +202.7 \\
\hline Left Rectus Femoris $(\boldsymbol{\mu} \mathbf{V})$ & 8.9 & 16.1 & +80.9 \\
\hline Left Vastus Lateralis $(\boldsymbol{\mu} \mathbf{V})$ & 12.7 & 33 & +159.8 \\
\hline Left Semitendinosus $(\boldsymbol{\mu} \mathbf{V})$ & 8.6 & 12 & +39.5 \\
\hline Left Biceps Femoris $(\boldsymbol{\mu} \mathbf{V})$ & 6.4 & 9.2 & +43.8 \\
\hline
\end{tabular}

semitendinosus and biceps femoris were $7.9 \mu \mathrm{V}$ and $3.7 \mu \mathrm{V}$ respectively. For the left leg the extension EMG values were $8.9 \mu \mathrm{V}$ for the rectus femoris and $12.7 \mu \mathrm{V}$ for the vastus lateralis. With the left leg flexion movement reporting values of $8.6 \mu \mathrm{V}$ for the semitendinosus and $6.4 \mu \mathrm{V}$ for the biceps femoris (Table 1).

After the four-week strength training intervention, post-test results revealed an increase of EMG activity by $3.6 \mu \mathrm{V}$ for the rectus femoris and $4.1 \mu \mathrm{V}$ for the vastus lateralis during the right leg extension movement. The left leg extension movement saw similar increases in EMG activity with the rectus femoris and vastus lateralis gaining increases of $7.22 \mu \mathrm{V}$ and $20.3 \mu \mathrm{V}$ respectively (Figure 1 ). The right leg flexion movement brought about an increase in $7.1 \mu \mathrm{V}$ for the semitendinosus and $7.5 \mu \mathrm{V}$ for the biceps femoris post-intervention. The left leg flexion movement also brought about increases in EMG activity by $4 \mu \mathrm{V}$ for the semitendinosus and $2.8 \mu \mathrm{V}$ for the biceps femoris post-intervention (Figure 2).

\section{Discussion}

This case study has demonstrated that a four-week resistance training intervention can significantly increase stability, lower-limb strength and muscular activity within an adult diagnosed with $\mathrm{CP}$. The length of the intervention and improved muscular activity across all muscle groups, suggests adaptations to the central nervous system may be main mechanism responsible for these changes. These findings implicate the need for further research using randomised clinical trials which explore the effects of resistance training in reducing falls-risk, whilst examining the physiological mechanisms that underpin these changes.

Statistically significant reductions were observed in total displacement, characterised by reductions in both mediolateral and anteroposterior displacement. The magnitude of this change will likely improve stability and the functioning of day-to-day activities. This suggests that seated quadricep and hamstring resistance training can improve static stability in adult CP patients in a little as four-weeks. The increase in peak torque observed in the left limb has been previously shown to correlate to mediolateral deviations in CoP during walking, which may be due to the quadriceps and hamstrings having secondary roles in mediolateral stabilisation. Specifically, improvements in hamstring strength which was observed in both left and right limbs, both of which displayed notably lower than normal function, will have significant benefits in limb support and activity. It is notable that the left limb showed improvements substantially greater than the right limb, which may be a result of the previous surgery carried out during early childhood. Importantly, it should be acknowledged that these improvements were much larger than would be expected in a four-week resistance intervention. The reduced force output observed in the right quadriceps may be a result of co-activation in the antagonist muscles which is commonly associated in $\mathrm{CP}$ patients when subjected to resistance training [19]. The impaired corticospinal tract function can lead to improper firing of excessive MUAPs to the antagonist muscles, leading to increased stiffness which ultimately restricts force output in the agonist muscle during maximal contraction [20].

An increase in muscular activity was observed across all muscle groups, suggesting possible strengthening of the efferent and afferent pathways of the central nervous system. This improvement is concurrent with increased force production and improved static stability. Although direct relationships cannot be assessed, it has been well established that CNS adaptations occur in an attempt to employ motor units more effectively in order to meet the demands of the resistance training 


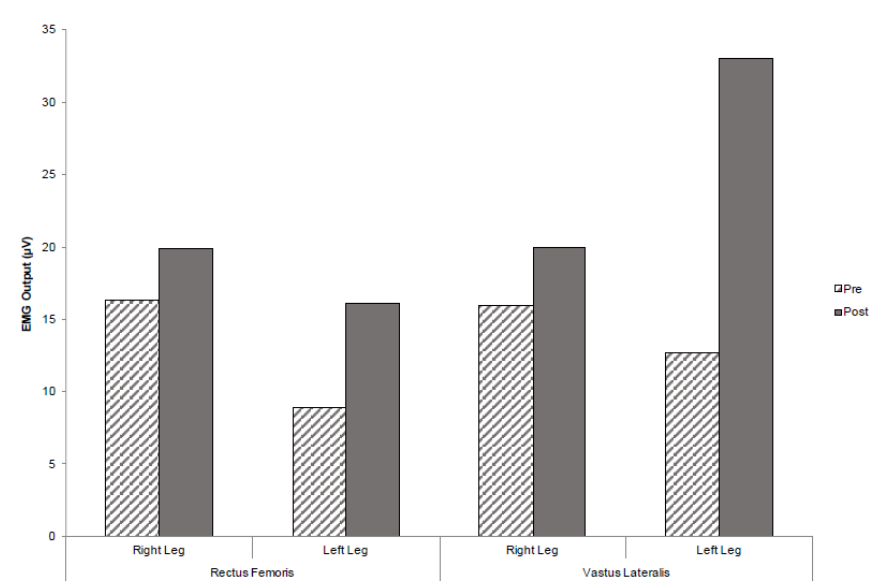

Figure 1. A graph to show mean EMG values for the rectus femoris and vastus lateralis muscle sites pre and post intervention, during the MVIC leg extension

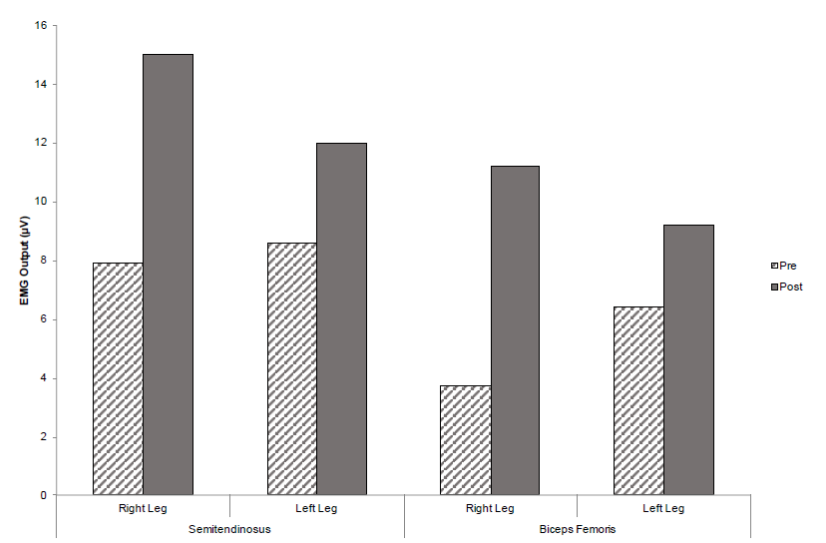

Figure 2. A graph to show mean EMG values for the semitendinosus and biceps femoris muscle sites pre and post intervention during the MVIC leg flexion

intervention [21]. This adds sufficient credibility to the application of EMG in measuring motor unit action potentials, when assessing the effectiveness of a resistance training intervention in CP patients. This evidence supports earlier studies that have outlined the importance of resistance training for improvements in muscular strength and functional activity in $\mathrm{CP}$ patients $[5,22,23]$.

The stimulation of the central nervous system may be the primary driver in the onset of adaptations, whereby resistance programmes should be designed accordingly. This is supported by Ahlborg, et al. [24] who carried out an 8-week whole-body vibration training (WBVT) intervention and a resistance training intervention in two separate groups. They found similar increases in gross motor function and muscle resistance measured at 30-degrees per-second in both groups. Whole body vibration training uses vibrations to stimulate both the muscle spindles and the alpha-motoneurons; this promotes the initiation of muscular contraction through the innervation of extrafusal muscle fibres. Although strength improvements gained through the use of WBVT have shown to have some inconsistencies [25], this research could provide an insight into the explanations behind these adaptations. Understanding the mechanisms that underpin these adaptations may direct practitioners in structuring resistance programmes to optimise improvements within adult CP patients. This is supported by Andersson, et al. [1] who reported significant improvements in isometric resistance and isokinetic concentric work, but no improvements in eccentric work. This may suggest that training programmes aimed at increasing metabolic stress, mechanical tension and muscular damage may not be appropriate for those diagnosed with CP.

This case study has provided evidence to suggest that increases in static stability occur concurrently with increased neuromuscular activity and force output as a result of a replicable four-week resistance training intervention. This suggests that resistance training programmes designed to increase strength and muscular activity of the lower-limbs are effective and may potentially reduce falls-risk in adult CP patients through improvements in static stability. Further randomised clinicaltrials are required to substantiate these claims and provide possible mechanistic explanations for these changes.

\section{Limitations}

Any research involving $\mathrm{CP}$ patients offers a range of problematic issues that may result in a detrimental effect on the validity and reliability of the research. The quantification of intensity has been arguably problematic in the in the confines of this case-study; the selection of an untrained subject although significant to the nature of this study and informative, has also presented some difficulty in the consistent monitoring of the working intensity of the subject. Verbal indicators were used throughout, with a simple RPE measure using a 1-10 scale, but given the unfamiliarity of the subject with the mode of exercise and loading, coupled with the length of the programme, the subject would require time to become accustomed to the physiological effects of training. Training load in the form of resistance applied by the participant was adjusted only once relative to the required intensity, which could negatively impact the maintenance of intensity and total volume throughout the duration of the programme [26]. A greater amount of baseline maximal strength tests, more frequent training sessions and a longer training period may help to compensate for any potential anomalies [27].

The use of EMG readings, although an effective gauge of muscular activity has some potential for abnormality due to the nature of the condition studied. It must be acknowledged that by nature, $\mathrm{CP}$ expresses itself as abnormal CNS activity and the data obtained, demonstrates a degree of variability in individual readings which could be considered significant [28]. Ultimately, a greater pre- and post-intervention period may have assisted to obtain more reliable baseline and post-testing results. This would not only affect the gains observed, but also the intensity at which the subject was working at.

\section{Practical applications}

This study provides evidence that the application of a resistance training intervention could be effective for adult $\mathrm{CP}$ patients in the form of spastic diplegia. This research suggests that even after the developmental years, a mature subject can still induce physiological and neural adaptations through the inclusion of resistance training. This is of particular importance for older subjects with the majority of their time spent in a wheelchair whereby processes such as osteopenia, osteoporosis and reduced bone mineral density are likely to be accelerated $[1,29]$. There is a clear need to expand upon this research using randomised clinical trials and larger cohorts. Future research should be directed towards investigating whether similar changes would be observed in dynamic stability and gross motor function [3040].

\section{Conclusion}

This case study suggests that a four-week resistance training intervention improves the static stability of adult CP patients, which may 
McManus A (2018) A 4-week resistance training intervention improves stability, strength and neuromuscular activity in the lower limb: A case study of a cerebral palsy adult

subsequently reduce fall-risk. Concomitant improvements in lowerlimb strength and muscle activation suggests central nervous system adaptations may be the primary cause of this improvement. This could provide those adult CP patients who have access to training facilities with an evidence-based approach to improving their condition. There is therefore a clear need to expand upon this research with more detailed exercise prescriptions and larger cohorts.

\section{References}

1. Andersson C, Grooten W, Hellsten M, Kaping K, Mattsson E (2003) Adults with cerebral palsy: walking ability after progressive strength training. Dev Med Child Neurol 45: 220-228. [Crossref]

2. Anderson MA, Laskin JJ (2010) Cerebral Palsy. In: ACSM's Resources for Clinical Exercise Physiology: Musculoskeletal, Neuromuscular, Neoplastic, Immunologic, and Hematologic Conditions. Meyers, J. and Nieman D, eds. Philadelphia, PA: Wolters Kluwer Health.

3. Carr JH, Shepherd RB, Ada L (1995) Spasticity: research findings and implications for intervention. Physiotherapy 8: 421-429.

4. Ratamess NA (2008) Adaptations to Anaerobic Training Programmes. In: Essentials of Strength Training and Conditioning. Baechle, T.R. and Earle, R.W, eds. Champaign IL: Human Kinetics.

5. Dodd KJ, Taylor NF, Damiano DL (2002) A systematic review of the effectiveness of strength-training programs for people with cerebral palsy. Arch Phys Med Rehabil 83: 1157-1164. [Crossref]

6. Guiliani CA (1991) Dorsal rhizotomy for children with cerebral palsy: support for concepts of motor control. Phys Ther 71: 248-259. [Crossref]

7. Scholtes VA, Becher JG, Comuth A, Dekkers H, Van Dijk L, et al. (2010) Effectiveness of functional progressive resistance exercise strength training on muscle strength and mobility in children with cerebral palsy: A randomised control trial. Dev Med Child Neurol 52: 107-113. [Crossref]

8. Franki I, Desloovere K, De Cat J, Feys H, Molenaers G, et al. (2012) The evidence-base for basic physical therapy techniques targeting lower limb function in children with cerebral palsy: A systematic review using the international classification of functioning, disability and health as a conceptual framework. J Rehabil Med 44: 385-395. [Crossref]

9. Park EY, Kim WH (2014) Meta-analysis of the effect of strengthening interventions in individuals with cerebral palsy. Res Dev Disabil 35: 239-249. [Crossref]

10. Damiano DL (2006) Activiity, activity, activity: rethinking our physical therapy approach to cerebral palsy. Phys Ther 86: 1534-1540. [Crossref]

11. Gorczynski P (2012) The use of single-case experimental research to examine physical activity, exercise, and physical fitness interventions: A review. J App Sport Psychol 25: 148-156.

12. Baechle TT, Earle RW, Wathen D (2008) Essentials of Strength Training and Conditioning. Champaign, IL: Human Kinetics.

13. Rosenbaum RPP, Walter S, Russell D, Wood E, Galuppi B (1997) Gross motor function classification system for cerebral palsy. Dev Med Child Neurol 39: 214-223.

14. Fox MD Reinbolt JA, Õunpuu S, Delp SL (2008) Mechanisms of improved knee flexion after rectus femoris transfer surgery. $J$ Biomech 42: 614-619. [Crossref]

15. Criswell E, Cram JR (2011) Cram's Introduction to Surface Electromyography. Sudbury, MA: Jones and Bartlett.

16. Suminski RR, Robertson RJ, Arslanian S, Kang J, Utter AC, et al. (1997) Perception of effort during resistance exercise. J Strength Condition Res 11: 261-265.

17. Ahtiainen JP, Pakarinen A, Alen M, Kraemer WJ, Häkkinen K (2005) Short Vs long rest period between the sets in hypertrophic resistance training: influence on muscle strength and size, and hormonal adaptations in trained men. J Strength Cond Res 19: 572-582. [Crossref]

18. Reid S, Hamer P, Alderson J, Lloyd D (2010) Neuromuscular adaptations to eccentric strength training in children and adolescents with cerebral palsy. Dev Med Child Neurol 52: 358-363. [Crossref]

19. Damiano DL, Martellotta TL, Sullivan DJ, Granata KP, Abel MF (2000) Muscle force production and functional performance in spastic cerebral palsy; relationship of cocontraction. Arch Phys Med Rehabil 81: 895-900. [Crossref]

20. Bohannon RW (1989) Is the measurement of muscle strength appropriate in patients with brain lesions? A special communication. Phy Ther 69: 452-457.
21. Gabriel DA, Kamen G, Frost G (2006) Neural adaptations to resistive exercise. Sports Med 36: 133-149. [Crossref]

22. McBurney H, Taylor NF, Dodd KJ, Graham HK (2003) A qualitative analysis of the benefits of strength training for young people with cerebral palsy. Dev Med Child Neurol 45: 658-663. [Crossref]

23. Taylor NF, Dodd KJ, Larkin H (2004) Adults with cerebral palsy benefit from participating in a strength training programme at a community gymnasium. Disabil Rehabil 26: 1128-1134. [Crossref]

24. Ahlborg L, Andersson C, Julin P (2006) Whole-body vibration compared with resistance training: effect on spasticity, muscle strength and motor performance in adults with cerebral palsy. $J$ Rehabil Med 38: 302-308. [Crossref]

25. de Ruiter CJ, van der Linden RM, van der Zijden MJ, Hollander AP, de Haan A (2003) Short term effects of whole-body vibration on maximal voluntary isometric knee extensor force and rate of force rise. Eur J Appl Physiol 88: 472-475. [Crossref]

26. Sweet TW, Foster C, McGuigan MR, Brice G (2004) Quantitation of resistance training using the session rating of perceived exertion method. J Strength Cond Res 18: 796802. [Crossref]

27. Allen J, Dodd KJ, Taylor NF, McBurney H, Larkin H (2004) Strength training can be enjoyable and beneficial for adults with cerebral palsy. Disabil Rehabil 26: 1121-1128. [Crossref]

28. Olsen JE, Ross SA, Foreman MH, Engsberg JR (2013) Changes in muscle activation following ankle strength training in children with spastic cerebral palsy: An electromyography feasibility case report. Phys Occup Ther Pediatr 33: 230-242. [Crossref]

29. Chad KE, Bailey DA, McKay HA, Zello GA, Snyder RE (1999) The effect of a weightbearing physical activity program on bone mineral content and estimated volumetric density in children with spastic cerebral palsy. J Pediatr 135: 115-117. [Crossref]

30. Aagaard P, Simonsen EB, Andersen JL, Magnusson P, Dyhre-Poulsen P (2002) Neural adaptation to resistance training: changes in evoked V-wave and H-reflex responses. $J$ Appl Physiol 92: 2309-2318. [Crossref]

31. Deschenes MR, Judelson DA, Kraemer WJ, Meskaitis VJ, Volek JS, et al. (2000) Effects of resistance training on neuromuscular junction morphology. Muscle Nerve 23: 1576-1581. [Crossref]

32. Dodd KJ, Taylor NF, Graham HK (2003) A randomized clinical trial of strength training in young people with cerebral palsy. Dev Med Child Neurol 45: 652-657. [Crossref]

33. Elder GC, Kirk J, Stewart G, Cook K, Weir D, et al. (2003) Contributing factors to muscle weakness in children with cerebral palsy. Dev Med Child Neurol 45: 542-550. [Crossref]

34. Fukunaga T, Roy RR, Shellock FG, Hodgson JA, Edgerton VR (1996) Specific tension of the human plantar flexors and dorsiflexors. J Appl Physiol 80: 158-165. [Crossref]

35. Levitt S (1977) Treatment of Cerebral Palsy and Motor Delay. Oxford: Blackwell Scientific Publications.

36. Pensini M, Martin A, Maffuletti MA (2002) Central versus peripheral adaptations following eccentric resistance training. Int J Sports Med 23: 567-574. [Crossref]

37. Sale DG, MacDougall JD (1981) Specificity in strength training: a review for the coach and athlete. Can J Appl Sport Sci 6, 87-92. [Crossref]

38. Scianni A, Butler JM, Ada L, Teixeira-Salmela LF (2009) Muscle strengthening is not effective in children and adolescents with cerebral palsy: A systematic review. Aust $J$ Physiother 55: 81-87. [Crossref]

39. Tedroff K, Knutson LM, Soderberg GL (2006) Synergistic muscle activation during maximum voluntary contractions in children with and without cerebral palsy. Dev Med Child Neurol 50: 377-381. [Crossref]

40. Thawrani D, Haumont T, Church C, Holmes L Jr, Dabney KW, et al. (2012) Rectus femoris transfer improves stiff knee gait in children with spastic cerebral palsy. Clin Orthop Relat Res 470: 1303-1311. [Crossref]

Copyright: (C)2018 McManus A. This is an open-access article distributed under the terms of the Creative Commons Attribution License, which permits unrestricted use, distribution, and reproduction in any medium, provided the original author and source are credited. 